\title{
Degeneration in the Zygapophysial Joint of the Fifth Lumbar Vertebra: The V-Shaped Sign Revealed by Bone Scintigraphy
}

\section{Xin-Li Xie* \\ Yan Liu* \\ Bing Cheng \\ Xiao-Guang Du \\ Qiao Ruan \\ Xing-Min Han}

Department of Nuclear Medicine, The First Affiliated Hospital of Zhengzhou University, Zhengzhou, 450052, Henan, People's Republic of China

*These authors contributed equally to this work.
Correspondence: Xing-Min Han Department of Nuclear Medicine, The First Affiliated Hospital of Zhengzhou University, No. I Of Jianshe East Road, Erqi District, Zhengzhou, 450052, Henan, People's Republic of China

Tel +8637166915504

Fax +86 371 66913059

Email hanxingmin_cn@।26.com
Objective: The aim of the study was to explore the nature of a V-shaped sign in the backbone of the fifth lumbar vertebra revealed by whole-body bone scintigraphy (WBBS).

Methods: A local single-photon emission computed tomography (SPECT) scan plus a computed tomography (CT) scan were performed on 41 patients in our department who had a V-shaped sign in the backbone of the fifth lumbar vertebra detected by WBBS. Image fusion was conducted to understand the manifestations of the changes in the V-shaped sign in the CT images in WBBS and to determine the nature of the lesion.

Results: All 41 patients presented with degenerative changes observed in the bilateral posterior zygapophysial joint of the fifth lumbar vertebra in the CT imaging bone window, bone hyperplasia of the articular process, joint surface hardening, and a joint gap. The vacuum sign could also be seen in some of these patients.

Conclusion: The typical V-shaped sign in the posterior zygapophysial joint of the fifth lumbar vertebra revealed by WBBS suggests degenerative changes in the zygapophysial joint of the fifth lumbar vertebra.

Keywords: lumbar, zygapophysial joints, degeneration, bone radionuclide imaging, MDP, single-photon emission computed tomography (SPECT)-CT, diagnosis

\section{Introduction}

Bone scintigraphy is the most sensitive method that can be used in the diagnosis of bone metastatic cancer. However, for diseases with single or few lesions, this method has a low qualitative value, as it is difficult to make an accurate diagnosis in such cases using bone scintigraphy. Hence, other imaging examinations are often used concurrently to verify the diagnosis. ${ }^{1}$ In this prospective study, single-photon emission computed tomography (SPECT)/computed tomography (CT) was performed on 41 patients, who presented with a V-shaped sign revealed by wholebody bone scintigraphy (WBBS). This was a special feature in the backbone of the fifth lumbar vertebra. This sign was confirmed to be a degenerative change in the posterior zygapophysial joint of the fifth lumbar vertebra.

\section{Patients and Methods}

\section{Patients}

This study involved 41 patients who had undergone WBBS. They had all done so to detect the presence of bone metastases. A V-shaped sign was found in the backbone 
of the fifth lumbar vertebra in all these patients. The study was conducted in accordance with the Declaration of Helsinki (as was revised in 2013). The study was approved by the ethics committee of the First Affiliated Hospital of Zhengzhou University. The patients were informed about the purpose of the study, and written consent was obtained from them.

\section{Imaging Methods}

The instrument used in this study was a Siemens Symbia T16 dual-detectors SPECT/CT system equipped with a low-energy high-resolution collimator. No special preparation was required before the examination. After being given an intravenous injection of $925-1110 \mathrm{MBq}$ of ${ }^{99} \mathrm{mTc}-$ methylene diphosphonate (MDP), the patients were asked to drink more water and to urinate as often as possible. Three hours later, they underwent anterior-toposterior and posterior-to-anterior systemic planar imaging. The scanner was guided by automatic tracking of the human body. The scanning speed was set at $15-20 \mathrm{~cm} /$ minute, and the matrix was set at $256 \times 1024$. After the end of the planar imaging, local SPECT plus CT was immediately performed on these patients, who had been found to have a V-shaped sign in the backbone of the fifth lumbar vertebra. First, X-ray positioning scanning was performed. Then, the scanning range was determined according to the positioning films. The fifth lumbar spine was set as the center of the scanning range, and the upper and lower boundaries were both $20 \mathrm{~cm}$ from the center. If the patient had other lesions, it was necessary to extend the range of the CT scanning. Then, CT transmission scanning was performed. The matrix was set at $256 \times 256$, and the thickness of the scanning slice was set at $5 \mathrm{~mm}$. After CT scanning, the examination table was automatically aligned, and SPECT acquisition was performed with the matrix set at $128 \times 128$. The acquisition was performed using a stepping method, with the step set at $6^{\circ}$ and acquisition time at $30 \mathrm{~seconds} /$ frame. The two detectors were each rotated $180^{\circ}$, and 64 images were acquired in total. The ${ }^{99} \mathrm{Mo}-{ }^{99} \mathrm{mTc}$ generator was provided by the China Institute of Atomic Energy, and the MDP was provided by the Jiangsu Institute of Nuclear Medicine.

\section{Image Reformation and Fusion}

The original SPECT projections were reformatted using the Flash 3D iterative reformation method; the subset number was four, the iteration number was eight, and the slice thickness was $3 \mathrm{~mm}$. A total of 128 frames of cross section images were acquired, and corresponding coronal, sagittal, and dynamic 3D images were also acquired. After reformation, the CT and SPECT images had the same matrix, number of pixels, and effective frame rate. The fusion of CT and SPECT images was achieved using Syngo workstation software.

\section{Image Analysis}

The WBBS diagnosis was performed by two nuclear medicine physicians, and the interobserver agreement for WBBS diagnosis for the two physicians was excellent, with an intraclass correlation coefficient (ICC) of 0.91 .

The SPECT/CT diagnosis was made jointly by a nuclear medicine physician and a CT doctor. The ICC of these two experts was 0.88 , also indicating an excellent interobserver agreement for SPECT/CT diagnosis.

\section{Results}

Forty-one patients were enrolled in this study. Among them, 10 patients were male and 31 patients were female, and the age range was 44-89 years, with an average age of $(62.95 \pm 11.02)$ years. Fourteen patients were diagnosed with lung cancer, 7 patients were diagnosed with breast cancer, and 3 patients were diagnosed with esophageal cancer. One patient was diagnosed with each of the following cancers: prostate cancer, renal carcinoma, multiplemyeloma, ovarian cancer, rectal cancer, pheochromocytoma, and parotid carcinoma. Eight patients were diagnosed with pain of an unknown origin, and 2 patients were diagnosed with unexplained systemic bone pain.

A V-shaped sign was found in the backbone of the fifth lumbar vertebra in all these patients (Figure 1). The specific manifestation was that in the posterior image of the WBBS, two high radioactive uptake regions were found in the bilateral posterior inferior edges of the fifth lumbar vertebra, with a shape similar to the letter V. Hence, it was called a V-shaped sign. WBBS combined with SPECT-CT fusion imaging confirmed that multiple bone metastases had occurred in 11 patients. Among these patients, 4 were diagnosed with breast cancer, 2 with lung cancer, 1 with rectal cancer, 1 with multiple-myeloma, 1 with prostate cancer, 1 with parotid carcinoma, and 1 with unexplained systemic bone pain. In addition, a definitive diagnosis could not be made using WBBS in 4 patients with a single or few lesions. These cases were finally diagnosed with SPECT-CT fusion imaging as being compression fractures. Using this approach, 2 patients were diagnosed with degenerative changes of the upper lumbar spine. For 


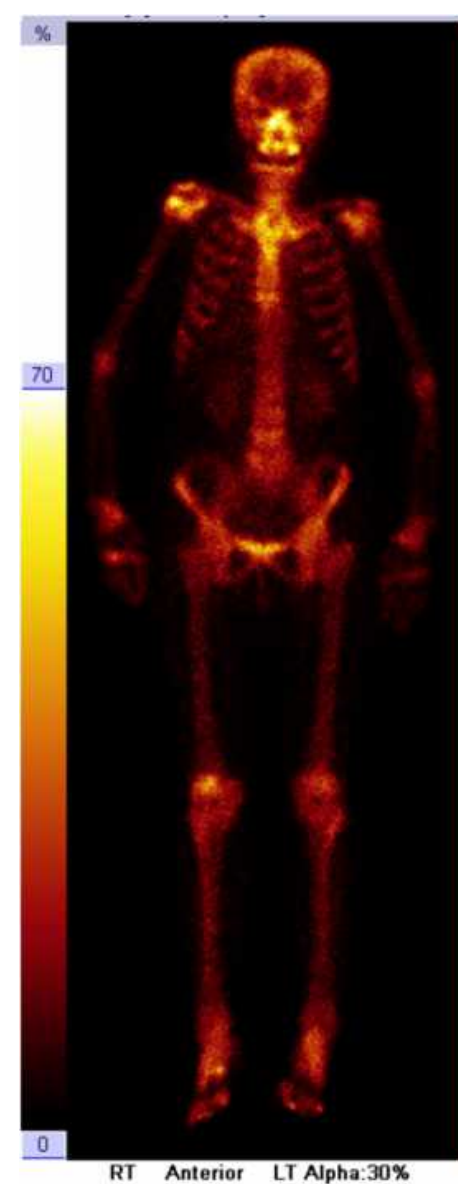

A

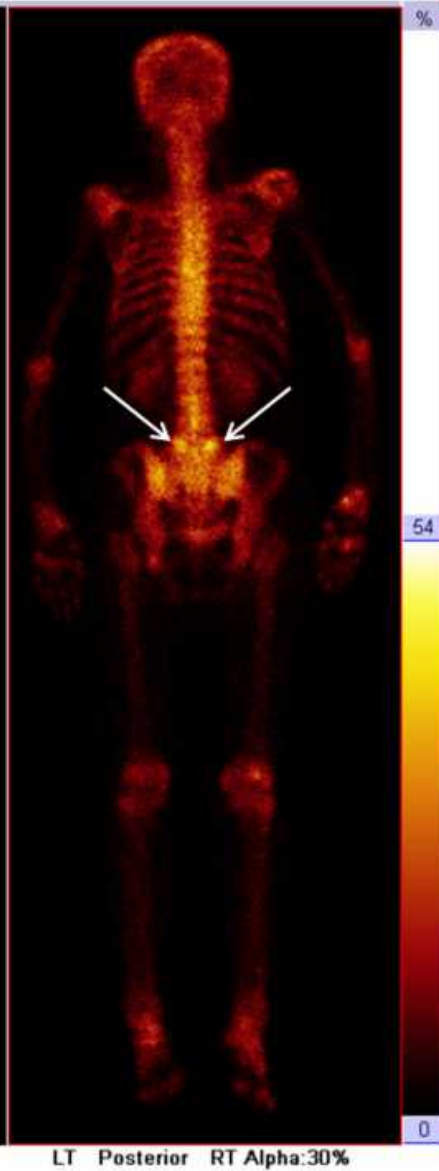

B

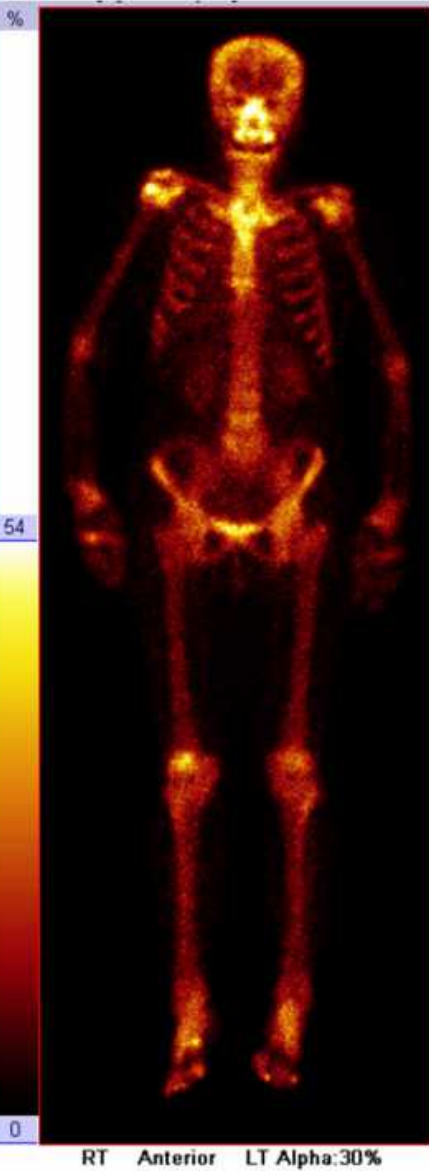

C

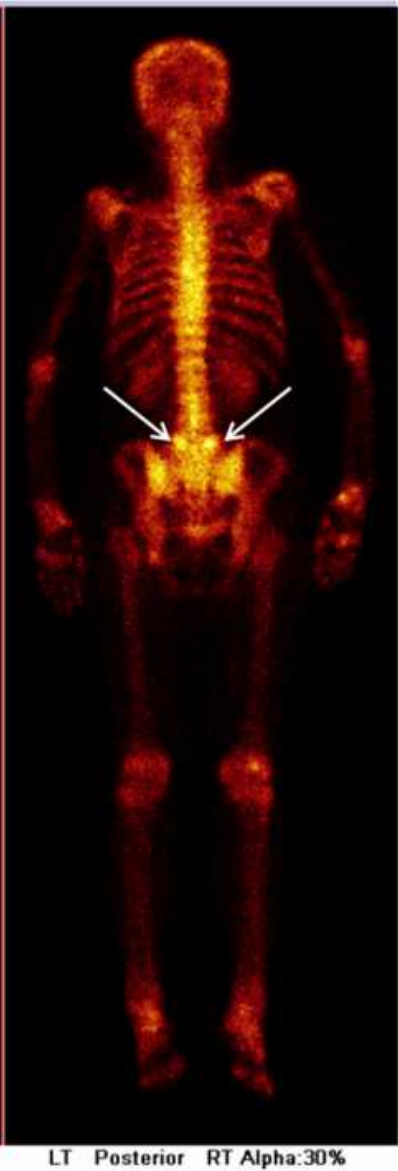

D

Figure I The whole body bone imaging in patients with typical performance: 55 years old female patient,the left breast cancer after I year,only found in the fifth lumbar back is "V-shaped sign" active focal bone metabolism,did not see signs of bone metastases. A: anterior view with low brightness; B: posterior view with low brightness; C: anterior view with high brightness; D: posterior view with high brightness

17 patients, the V-shaped sign in the backbone of the fifth lumbar vertebra was only revealed by WBBS, while the other approaches revealed no findings. Among these 17 patients, 7 were diagnosed with lung cancer, 2 with breast cancer, 1 with renal carcinoma, 1 with ovarian cancer, 1 with esophageal cancer, 1 with pheochromocytoma, and 4 patients were diagnosed with pain of an unknown origin.

In the $\mathrm{CT}$ images, the $\mathrm{V}$-shaped sign in the fifth lumbar vertebra in all 41 patients manifested as degenerative changes in the bilateral posterior zygapophysial joint. These changes included the following: (1) bone hyperplasia of the small articular process, lip-shaped bone hyperplasia was found in the joint process when severe facet hypertrophy could be observed and osteophytes had formed; (2) a change in the joint cavity, the joint cavity became narrower or broader, the articular surface hardened, and the vacuum sign was found in the joint cavity in some of the patients (Figure 2). Figures 1 and 2 involve the same patient.

\section{Discussion}

In the clinical diagnosis of WBBS, we often encounter the problem that a qualitative diagnosis cannot be established for highly active metabolic foci in certain bones in the body. This is the objective circumstance in WBBS. The sensitivity of WBBS in the diagnosis of bone metastases is very high. Most research has reported that the sensitivity of ${ }^{99} \mathrm{mTc}-\mathrm{MDP}$ bone scintigraphy in the diagnosis of bone metastases is the same as that of fluorodeoxyglucosepositron emission tomography, ${ }^{2-10}$ and it is significantly higher than that of X-ray and CT examinations. The sensitivity of X-ray examinations is approximately $57.0 \%$, while the sensitivity of CT is approximately $67.4 \% .{ }^{11}$ In general, these lesions can be found 3-6 months, or even up 


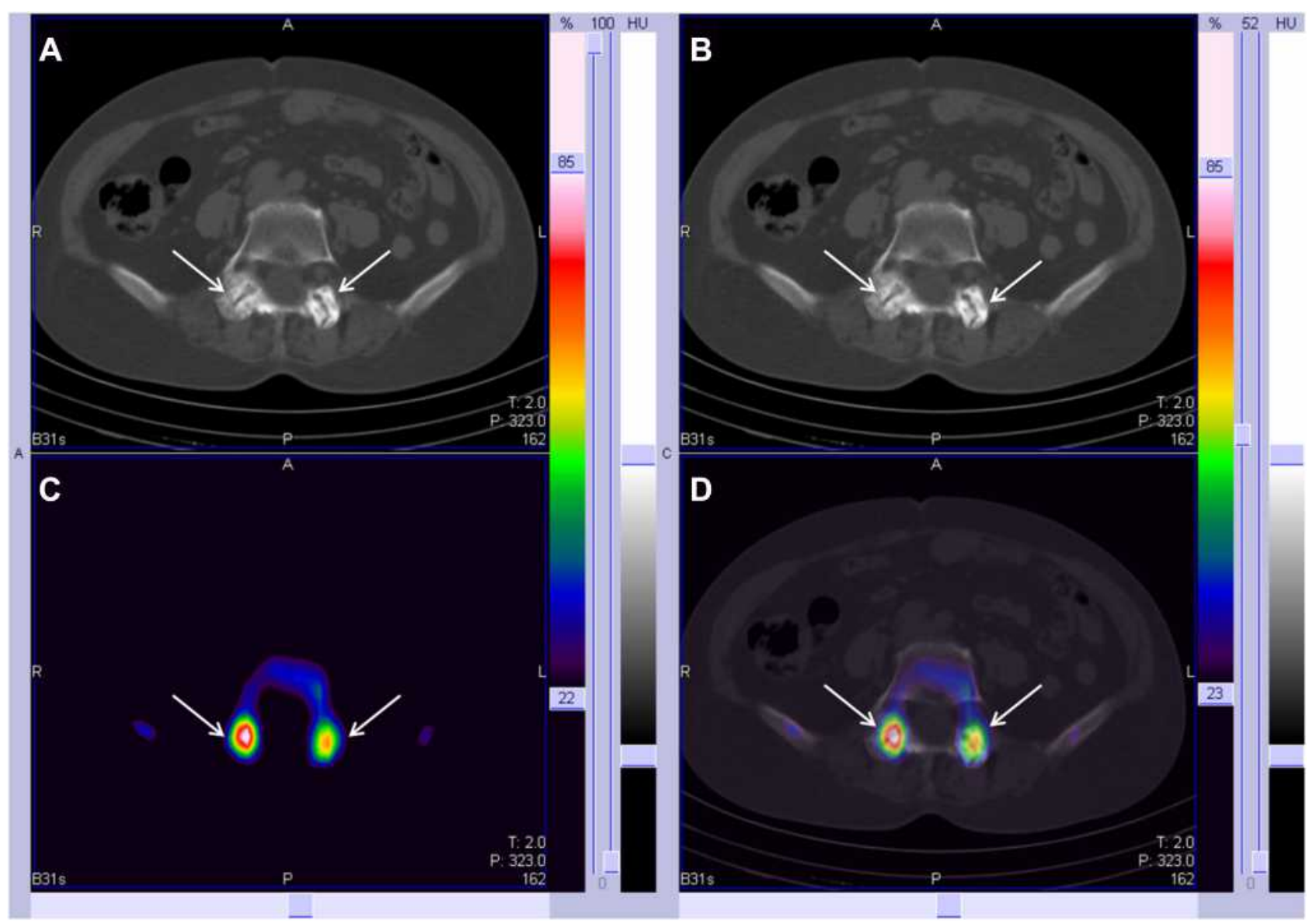

Figure 2 Patients with typical CT performance. A and B: CT continuous slices in bone window; C: SPECT cross section imaging; D: SPECT/CT fusion imaging.

to 18 months, earlier than they can with X-ray examinations. ${ }^{11}$ The sensitivity of magnetic resonance imaging (MRI) is also high, up to $88 \%$, but its cost is also high, and the scanning range is limited. ${ }^{12}$ Therefore, WBBS is the most sensitive and widely clinically-used imaging approach in the diagnosis of bone metastases. However, the specificity of WBBS, which is approximately $40-50 \%$ in clinical reports, is poor. The positive information revealed by WBBS only reflects the abnormity of metabolism in bone tissue, but benign and malignant lesions of bones such as hemangioma, bone hyperplasia, bone tuberculosis, bone tumors, and bone metastases can all present with this change. Therefore, when establishing a diagnosis using WBBS, it should be closely combined with the medical history of patients and with other imaging examinations, if necessary, such as CT and MRI. ${ }^{1,13}$ The low diagnostic specificity of WBBS dictates that in clinical practice, when we encounter the manifestation of uncharacteristic bone metastases, such as single or few lesions, it is difficult to establish a clear qualitative diagnosis. Since many benign lesions present with the manifestation of highly concentrated radioactivity, benign lesions cannot be distinguished by differences in their characteristics.

In the previous clinical diagnosis of WBBS, whenever the manifestation of typical multiple bone metastases was encountered combined with the V-shaped sign in the back bone of the fifth lumbar vertebra, little attention was paid to the local V-shaped sign because multiple bone metastases had already been definitively diagnosed. For the manifestation of the V-shaped sign in the backbone of the fifth lumbar vertebra alone, since WBBS cannot enable a qualitative diagnosis of a single lesion, it is often just described as high bone metabolism found in the fifth lumbar vertebra. Since it is a single lesion occurring in the lower part of the lumbar vertebrae, it is highly possible 
that this lesion is benign, and it is recommended that clinical tests or other imaging examinations should be used together. However, clinicians or patients often ignore this when they arrive at such a result, since they do not consider bone metastases. The author encountered 41 patients with the V-shaped sign in the backbone of the fifth lumbar vertebra through clinical WBBS diagnosis. Due to the application of the SPECT-CT imaging system in recent years, images can now be fused on the same machine, which has significantly improved the positioning and qualitative diagnosis value of single or few lesions. ${ }^{14}$ We carried out the analysis of the V-shaped sign combined with CT images in these 41 patients, and the results indicated that these were all degenerative changes in the zygapophysial joint in the back bone of the fifth lumbar vertebra.

Bone metastases occur very often in the vertebrae but not so often in the posterior vertebral facet joints and the spinous processes. In typical bone metastases, high radioactive uptake regions are often found on one side or in the whole vertebra. In particular, in the analysis of WBBS images combined with CT images, the high radioactive uptake region in one side of the vertebral body often presents with osteolytic lesion shadow, local soft tissue shadow, or high density osteogenesis metastasis shadow. Furthermore, the V-shaped sign occurs in the bilateral posterior inferior margins of the vertebral body, which is significantly different from typical vertebral metastases. On the fusion images of SPECT/CT on the same machine fusion image, the typical changes of degeneration can be found in the posterior vertebral facet joints.

The lumbar zygapophysial joint is also called the lumbar facet joint or intervertebral facet joint. It is composed of the upper and lower articular processes in the pedicle of the vertebral arch that connects with the vertebral lamina. The articular surface of the upper articular process faces the rear inner side, and the articular surface of the lower articular process faces the anterior side. The joint space of the right and left zygapophysial joints shows a V shape when viewed from the back. At home and abroad, studies have found that the degeneration of the lumbar intervertebral disc and facet joint is the basis of the degeneration of the function of the vertebral column. Due to the longterm effect of narrowing the height of the intervertebral space in the same segment or accumulation of the strain, the lumbar facet joint will undergo degenerative changes, such as articular cartilage exfoliation, bone softening, an uneven surface, facet joint hyperplasia plus osteophyte formation, and joint space narrowing or broadening. ${ }^{15}$ These changes in the bones increase the metabolism of the local bones. Therefore, it manifests as local radioactive aggregation in WBBS images, exhibiting a V-shaped change when viewed from the back.

Degenerative changes of the zygapophysial joint can compress the spinal cord or nerve root. The zygapophysial joint is dominated by the posterior ramus of the spinal nerve. Compression and traction of the nerve can cause lower back pain. In this study, the average age of these 41 patients was 62.95 years old. The author considered that these were all degenerative changes of the lumbar zygapophysial joint caused by long-term strain. Furthermore, 25 patients complained of lumbago.

WBBS is widely applied in the clinical diagnosis of multiple bone metastases due to its high sensitivity. However, its qualitative value in the diagnosis of only a few lesions is not high due to the lack of characteristics. CT, MRI, and other imaging tests are often used concurrently to determine a clear diagnosis. Therefore, it is worth summarizing the features of benign lesions in bone scintigraphy images to improve the diagnostic specificity of bone scintigraphy in the diagnosis of bone diseases. In this study, the author believes that an understanding of the $\mathrm{V}$-shaped sign, as well as the degenerative change of characteristic performance of bone scintigraphy on the lumbar facet joint, should be of some guidance for clinical practice, as well as making it unnecessary for patients to have other imaging tests and saving medical resources.

\section{Conclusions}

In this study, our results showed that the typical V-shaped sign in the posterior zygapophysial joint of the fifth lumbar vertebra revealed by WBBS suggested degenerative changes in the zygapophysial joint of the fifth lumbar vertebra.

\section{Acknowledgments}

We are particularly grateful to all the people who have given us help on our article.

\section{Funding}

There is no funding to report.

\section{Disclosure}

The authors declare that they have no conflicts of interest. 


\section{References}

1. Al-Bulushi NK, Abouzied ME. Comparison of 18F-FDG PET/CT scan and $99 \mathrm{mTc}-\mathrm{MDP}$ bone scintigraphy in detecting bone metastasis in head and neck tumors. Nucl Med Commun. 2016;37:583-588. doi:10.1097/MNM.0000000000000479

2. Minamimoto R, Loening A, Jamali M, et al. Prospective comparison of 99mTc-MDP scintigraphy, combined 18F-NaF and 18F-FDG PET/ $\mathrm{CT}$, and whole-body MRI in patients with breast and prostate cancer. J Nucl Med. 2015;56:1862-1868. doi:10.2967/jnumed.115.162610

3. Agarwal KK, Mukherjee A, Sharma P, Bal C, Kumar R. Incremental value of $99 \mathrm{mTc}-\mathrm{MDP}$ hybrid SPECT/CT over planar scintigraphy and SPECT in avascular necrosis of the femoral head. Nucl Med Commun. 2015;36:1055-1062. doi:10.1097/MNM.0000000000000357

4. Sahin E, Zincirkeser S, Akcan AB, Elboga U. Is (99m)Tc-MDP whole body bone scintigraphy adjuvant to (18)F-FDG-PET for the detection of skeletal metastases? J BUON. 2014;19:291-296.

5. Yang Z, Zhang Y, Shi W, et al. Is $18 \mathrm{~F}-F D G$ PET/CT more reliable than $99 \mathrm{mTc}-\mathrm{MDP}$ planar bone scintigraphy in detecting bone metastasis in nasopharyngeal carcinoma? Ann Nucl Med. 2014;28:411-416. doi:10.1007/s12149-014-0831-z

6. Byun BH, Kong CB, Lim I, et al. Comparison of (18)F-FDG PET/CT and $(99 \mathrm{~m}) \mathrm{Tc}-\mathrm{MDP}$ bone scintigraphy for detection of bone metastasis in osteosarcoma. Skeletal Radiol. 2013;42:1673-1681. doi:10.1007/ s00256-013-1714-4

7. Chakraborty D, Bhattacharya A, Mete UK, Mittal BR. Comparison of $18 \mathrm{~F}$ fluoride PET/CT and 99mTc-MDP bone scan in the detection of skeletal metastases in urinary bladder carcinoma. Clin Nucl Med. 2013;38:616-621. doi:10.1097/RLU.0b013e31828da5cc

8. Jiang L, Han L, Tan H, Hu P, Zhang Y, Shi H. Diagnostic value of ${ }^{99} \mathrm{mTc}-\mathrm{MDP}$ SPECT/spiral CT in assessing indeterminate spinal solitary lesion of patients without malignant history. Ann Nucl Med. 2013;27:460-467. doi:10.1007/s12149-013-0707-7
9. Damle NA, Bal C, Bandopadhyaya GP, et al. The role of $18 \mathrm{~F}$-fluoride PET-CT in the detection of bone metastases in patients with breast, lung and prostate carcinoma: a comparison with FDG PET/CT and 99mTc-MDP bone scan. Jpn J Radiol. 2013;31:262-269. doi:10.1007/s11604-013-0179-7

10. Sharma P, Agarwal KK, Kumar S, et al. Utility of (99m)Tc-MDP hybrid SPECT-CT for diagnosis of skull base osteomyelitis: comparison with planar bone scintigraphy, SPECT, and CT. Jpn J Radiol. 2013;31:81-88. doi:10.1007/s11604-012-0148-6

11. Chen XL, Li Q, Cao L, Jiang SX. Diagnostic role of (99)Tc(m)-MDP SPECT/CT combined SPECT/MRI Multi modality imaging for early and atypical bone metastases. Int J Clin Exp Med. 2014;7:5336-5341.

12. Chang CY, Gill CM, Joseph Simeone F, et al. Comparison of the diagnostic accuracy of $99 \mathrm{~m}$-Tc-MDP bone scintigraphy and 18 F-FDG PET/CT for the detection of skeletal metastases. Acta Radiol. 2016;57:58-65. doi:10.1177/0284185114564438

13. Pires AO, Borges US, Lopes-Costa PV, Gebrim LH, da Silva BB. Evaluation of bone metastases from breast cancer by bone scintigraphy and positron emission tomography/computed tomography imaging. Eur J Obstet Gynecol Reprod Biol. 2014;180:138-141. doi:10.1016/j.ejogrb.2014.06.021

14. Shen G, Zhou L, Jia Z, Zhang W, Wang Q, Deng H. [Diagnostic value of MRI versus 99Tcm-MDP bone scan in osseous metastasis of prostate cancer: a meta-analysis]. Sheng Wu Yi Xue Gong Cheng Xue Za Zhi. 2014;31:881-887. Chinese.

15. Yu Y, Kuang A. [The diagnostic value of $99 \mathrm{mTc}-\mathrm{MDP}$ bone scan and computed tomography for bone metastases of breast cancer: a systematic review]. Sheng Wu Yi Xue Gong Cheng Xue Za Zhi. 2014;31:552-557. Chinese.
International Journal of General Medicine

\section{Publish your work in this journal}

The International Journal of General Medicine is an international, peer-reviewed open-access journal that focuses on general and internal medicine, pathogenesis, epidemiology, diagnosis, monitoring and treatment protocols. The journal is characterized by the rapid reporting of reviews, original research and clinical studies

\section{Dovepress}

across all disease areas. The manuscript management system is completely online and includes a very quick and fair peer-review system, which is all easy to use. Visit http://www.dovepress.com/ testimonials.php to read real quotes from published authors. 